\title{
Design of Components and Circuits Underneath Integrated Inductors
}

\author{
Frank Zhang, Student Member, IEEE, and Peter R. Kinget, Senior Member, IEEE
}

\begin{abstract}
In this paper, we present a study of the eddy current effect of devices placed underneath and inside an on-chip inductor. We verified the performance of such area-saving structures through electromagnetic (EM) simulations and measurement of test structures. We used layout techniques to minimize eddy current loss and magnetic coupling between the devices and the inductor, and constructed a complete voltage-controlled oscillator (VCO) inside an inductor. Measurement results show that this compact VCO has an equal performance in phase noise and output power as compared to a traditional VCO while reducing the area by about $50 \%$. The techniques presented in this paper are general and can be implemented in most layouts without extra post-processing steps.
\end{abstract}

Index Terms-Eddy current, layout, metal fill, radio frequency integrated circuits (RFIC), voltage-controlled oscillator (VCO).

\section{INTRODUCTION}

CPIRAL on-chip inductors are found in many radio frequency (RF) transceivers and are the essential components for low noise amplifiers, filters, voltage-controlled oscillators (VCO), and other RF building blocks. On-chip inductors are indispensable in RF circuits because their alternatives use active components which are noisy and consume too much power. However, on-chip inductors have the drawback of consuming large die area, which translates to higher cost. An inspection of existing RF integrated circuit (RFIC) layouts (for example, the GPS receiver shown on the cover of [1] and the die photo of a transceiver in [2]) indicates that the on-chip inductors used in the RF sections of the integrated circuits dominate the die area of the chip. This trend will become even more significant in future process nodes since passive devices do not significantly reduce in size with process geometry scaling in contrast to their active counterparts. Up to now, the real estate underneath the inductors has not been utilized because of the concern that components under the inductor would degrade the quality factor, $Q$, of the inductor through eddy current loss. However, if the size of the devices placed in and around the inductor is kept small, the induced eddy current loops are localized in small regions which keeps the losses to a minimum. Furthermore, by carefully planning the current paths of the devices, magnetic coupling between the device currents and the inductor currents can be reduced. With these two properties in mind, we explore

Manuscript received December 15, 2005; revised April 24, 2006. This work was supported in part by the National Science Foundation under NSF MRI Grant ECS-03-20666 for test equipment. The work of F. Zhang was supported through an NSF GK-12 fellowship.

The authors are with the Department of Electrical Engineering, Columbia University, New York, NY 10027 USA.

Digital Object Identifier 10.1109/JSSC.2006.881547

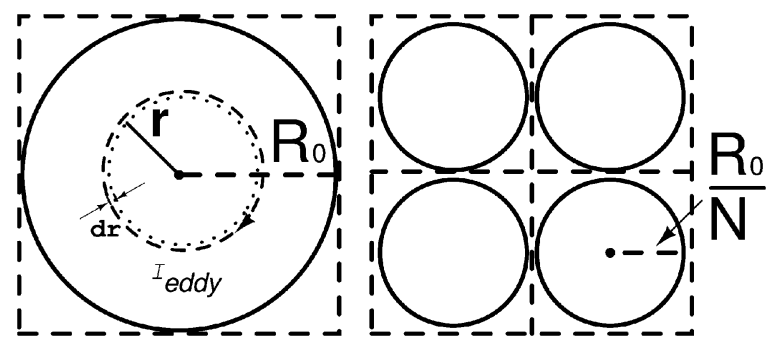

Fig. 1. A thought experiment demonstrating the reduction of eddy current loss in metal fill cells through the localization of eddy current loops inside smaller surface areas.

the possibility of placing active and passive devices underneath an on-chip inductor, and thus utilizing the third dimension of an integrated circuit to reduce the die area and the cost of RFICs. In Section II, we investigate the effect of metal fills placed in and around the inductor on its $Q$ using electromagnetic (EM) simulations and measurements on test structures. In Section III, we replace metal fills with multiple small varactors inside the inductor to study the tunability and the $Q$ of the resulting resonator. Finally, in Section IV, we add circuitry that provides a negative resistance to the resonator, thus forming a VCO. We use two identically designed VCOs but with different layouts to verify the proposed "circuit-in-the-coil" concept. The VCO-in-the-coil [3] has a VCO tank layout which places the transistors and varactors under the inductor, but avoids any significant additional losses. We compare the measured performance of this compact $\mathrm{VCO}$ with a VCO with a standard layout to demonstrate the effectiveness of the proposed approach.

\section{Metal Fill Test Structures}

Modern fabrication processes require as uniform a metal density as possible, and one potential motivation for utilizing the area underneath an inductor is to increase metal density. On-chip inductors are large, but they are often made of only two metal layers. Placing metal fills inside or near the inductor can increase the metal density count for all metals, but has the possible drawback of decreasing the $Q$ of the inductor, through eddy current loss. Measurement results of inductors with metal fills have been reported before [4]-[6], but without establishing a relationship between fill cell size and inductor $Q$. This relationship is of great interest to this work since it allows us to estimate the largest device that can be placed inside an inductor.

Fig. 1 illustrates a thought experiment demonstrating eddy current in metal fills inside a magnetic field. Since we are mainly interested in the effect of metal fill structures with small dimensions which introduce minimal extra losses, we assume that the 


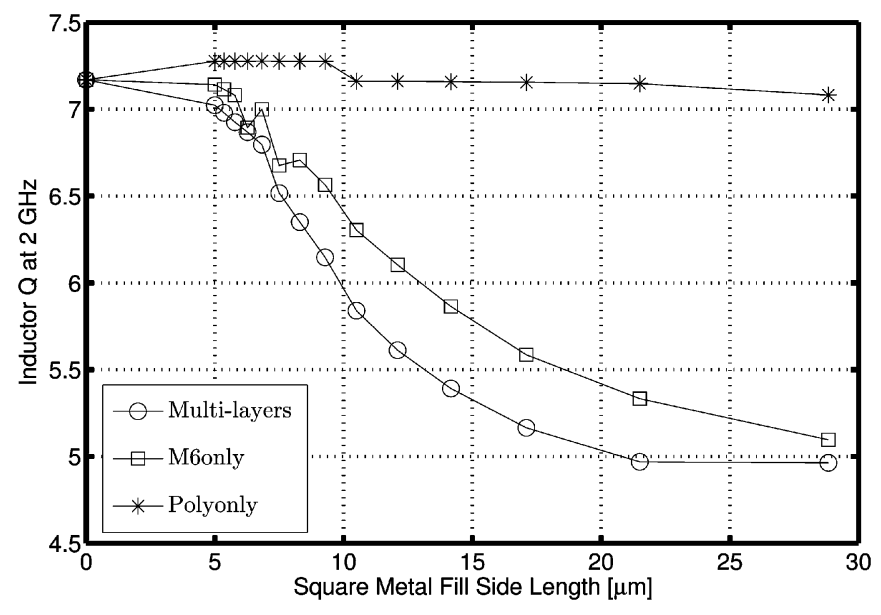

Fig. 2. Simulated inductor $Q$ from EM simulator for different metal fill cell sizes and resistivity. Metal fill side length of zero represents the case where no metal fills were used.

skin effect in the metal fill and the effect of the induced currents on the magnetic field can be neglected. The large circular metal fill in Fig. 1 has a radius $R_{0}$, and occupies about the same amount of area as four smaller circular metal fills with radius $R_{0} / N$, where $N=2$ in this case. For mathematical convenience, we use circular metal fills to approximate square metal fills used in real layouts. Assuming a small fill cell size compared to the dimensions of the inductor, the magnetic field $B$ is uniform over the area of the metal fill cell, and is not a function of the distance from the center of the fill cell, $r$. The magnetic flux $\Phi$, enclosed in a circular loop of radius $r$, is given by [7]

$$
\Phi=\oint B \cdot d A=B \cdot\left(\pi r^{2}\right) .
$$

Using Faraday's Law,

$$
V=-\frac{d \Phi}{d t}=-\frac{d B}{d t} \cdot \pi r^{2}
$$

where $V$ is the potential developed along any current path as a result of changing flux induced by the $\mathrm{AC}$ current in the inductor. The negative sign indicates that the current $I_{\text {eddy }}$, induced by $V$, will flow in such a direction as to oppose the flux that produced it. The resistance $R$ of a thin cylindrical sheet of metal fill, bounded by the dashed and dotted lines in Fig. 1, is equal to

$$
R=\frac{\rho}{h \cdot d r} \cdot(2 \pi r)
$$

where $\rho$ is the resistivity of the fill metal, $h$ is the height of the metal fill, and $d r$ is the incremental thickness of the cylindrical sheet. From (2) and (3), the total power dissipated in a metal fill with radius $R_{0}$ is

$$
P_{\text {diss }}=\int \frac{V^{2}}{R}=\int_{0}^{R_{0}}\left(\frac{d B}{d t}\right)^{2} \cdot \frac{\pi \cdot h}{2 \cdot \rho} \cdot r^{3} d r
$$

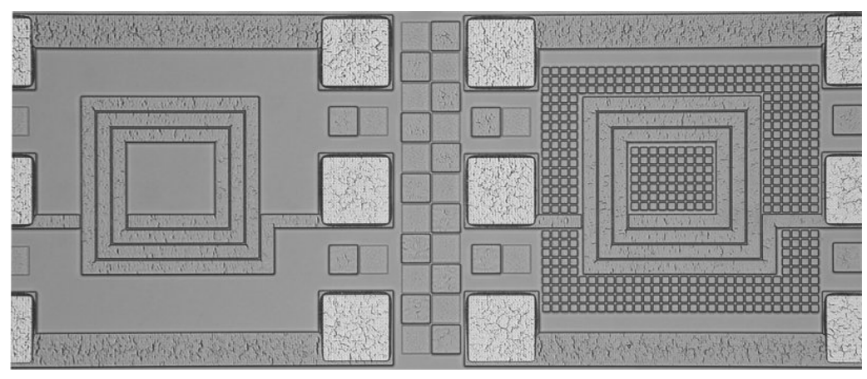

Fig. 3. Die photo of the test structures to evaluate the effect of metal fills on inductors: plain inductor (left), inductor with metal fills (right).

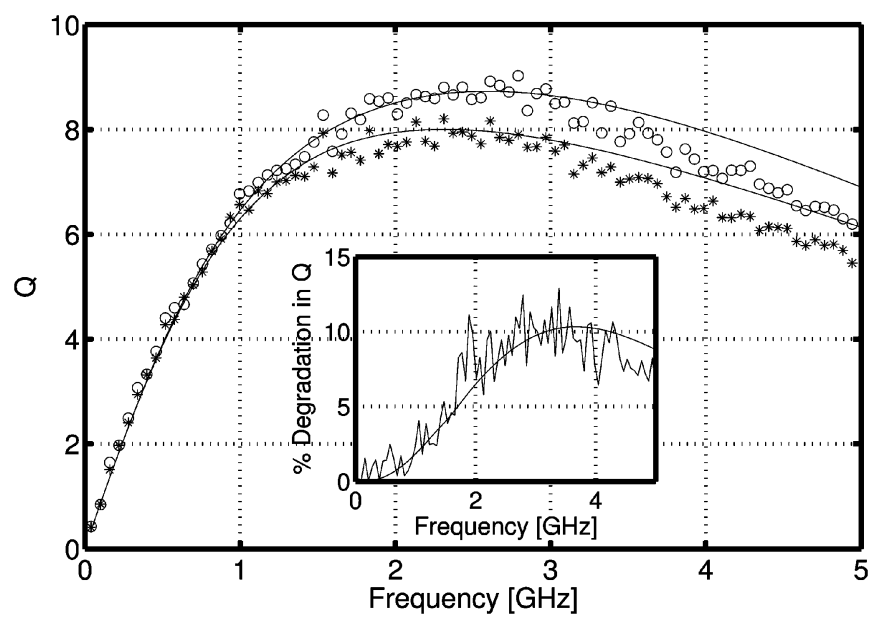

Fig. 4. Measured $Q$ of a plain inductor (o) and an inductor with metal fills (*), and corresponding simulation results (-). Inset: Percentage degradation in $Q$ with respect to frequency.

Since a large metal fill can be replaced by $N^{2}$ small metal fills, the power dissipation for the two cases are

$$
P_{\text {diss }}= \begin{cases}\left(\frac{d B}{d t}\right)^{2} \cdot \frac{\pi \cdot h}{8 \cdot \rho} \cdot R_{0}^{4} & \text { (one large fill cell) } \\ \left(\frac{d B}{d t}\right)^{2} \cdot \frac{\pi \cdot h}{8 \cdot \rho} \cdot \frac{R_{0}^{4}}{N^{2}} & \left(N^{2}\right. \text { small fill cells). }\end{cases}
$$

The power dissipated in the metal fills is an additional loss mechanism for the inductor and thus reduces its $Q$. However, (5) illustrates that this extra loss can be significantly reduced by using metal fills with small fill cell sizes. The equations in (5) provide guidelines on device sizing inside an inductor but are not sufficiently accurate for quantitative estimates. We ran extensive full-wave simulations on inductors with different fill cell sizes using the EM simulator EMX [8]. Fig. 2 shows the simulated $Q$ at $2 \mathrm{GHz}$ of a five-turn 4.5-nH differential inductor, $L_{\text {diff }}$, for different fill cell sizes and resistivity. The inductor $L_{\text {diff }}$ is used in the resonator and VCO circuits described in later sections. The inductor is constructed using the thick top metal, and has an outer diameter of $200 \mu \mathrm{m}$, inner diameter of $80 \mu \mathrm{m}$, trace width of $10 \mu \mathrm{m}$, and a trace spacing of $3 \mu \mathrm{m}$. The metal fills are constructed by stacking all the available metal and polysilicon layers without vias in between, and they are placed in the center of the inductor. Two important observations can be made from Fig. 2 which act as design guidelines for the designs of the 

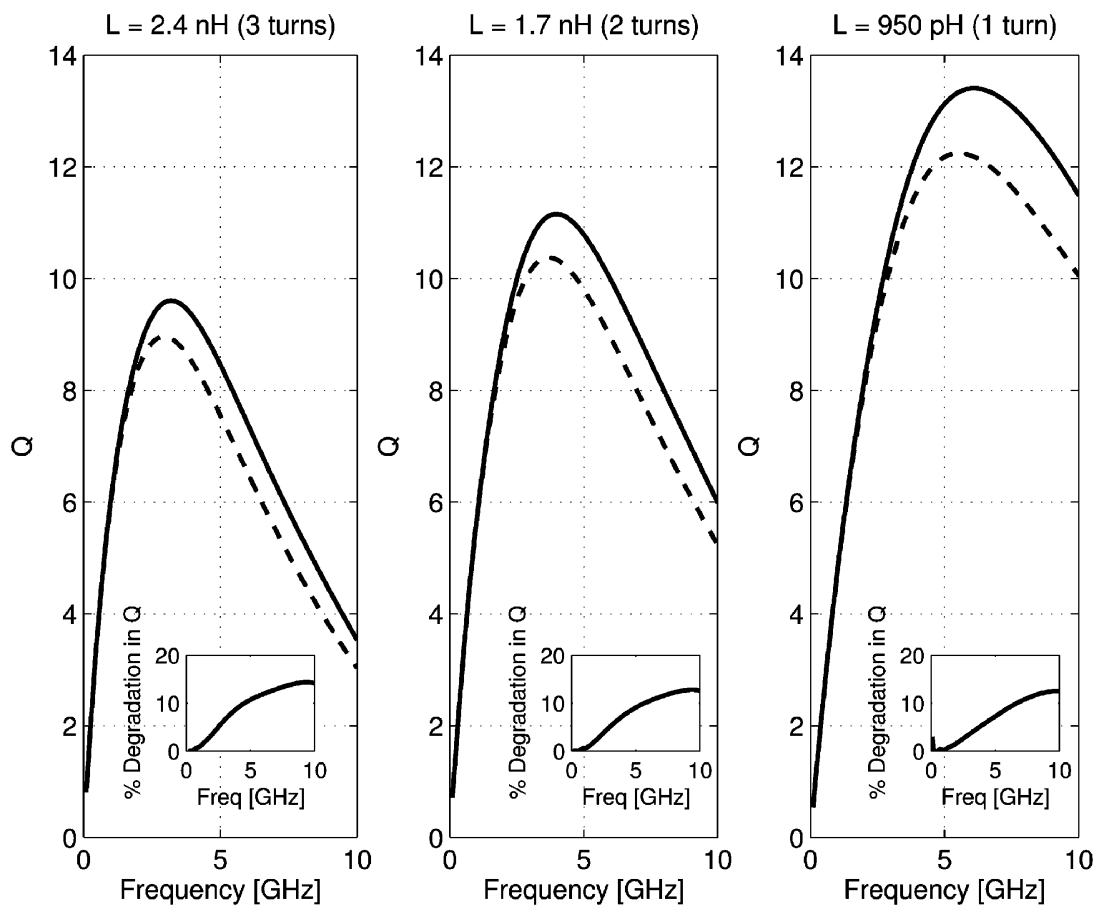

Fig. 5. Simulated $Q$ of inductors with (-) and without (- -) metal fills for different number of turns. Inset: percentage degradation in $Q$ with respect to frequency.

latter sections. For higher resistivity material, such as polysilicon, the eddy current loss is small, as predicted by (5). This implies, for example, polysilicon capacitors can be placed underneath the inductor without significantly degrading the $Q$ of the inductor. For low resistivity material, such as metal fills, the degradation on the $Q$ of the inductor depends on the area of the metal fill. For metal dimension larger than $7 \mu \mathrm{m}$ by $7 \mu \mathrm{m}$, the $Q$ of the inductor degrades rapidly, but for area less than that, they can be placed under the inductor without causing significant $Q$ degradation. $7 \mu \mathrm{m}$ by $7 \mu \mathrm{m}$ is large enough of an area for many practical devices.

The accuracy of EMX simulation was compared against measurement data from test structures in a $0.25-\mu \mathrm{m}$ BiCMOS process. The die photo of the test structures is shown in Fig. 3. A single-ended 2.3-nH inductor was constructed using the thick top metal layer, with an outer diameter of $200 \mu \mathrm{m}$, an inner diameter of $100 \mu \mathrm{m}$, a trace width of $10 \mu \mathrm{m}$, and a trace spacing of $3 \mu \mathrm{m}$. The metal fills were $7 \mu \mathrm{m}$ by $7 \mu \mathrm{m}$ squares with a spacing of $3 \mu \mathrm{m}$, and were of the multi-layer type. The metal fills were placed both inside and around the inductor. Fig. 4 shows the simulated and measured $Q$ of the test inductors. A small error of less than 5\% in $Q$ was observed between the simulated and the measured data below $3 \mathrm{GHz}$.

A maximum $Q$ degradation of about $10 \%$ occurs at its peak, similar to results reported in [5] and [6]. The typical application range of an inductor in a tunable VCO is below its peak $Q$ frequency since the varactors and the parasitics of the active devices add significantly to the tank capacitance [9].

To illustrate the effect of the metal fills on different size inductors, we removed the inner turns of the calibrated inductor discussed above. For the same area, fewer turns in a inductor results in lower inductance and higher $Q$. Using EM simulations, we compared the $Q$ of three different inductors with and without metal fills inside. Fig. 5 shows that the relative degradation in $Q$ due to metal fills is the same for inductors with different $Q \mathrm{~s}$.

\section{Resonator Test StRUCtURES}

Placing metal fills inside an inductor is not as useful as placing devices that would otherwise occupy area outside of the inductor. Therefore, next we replaced the metal fills with varactors. Eighty-two small varactors of dimension $12 \mu \mathrm{m}$ by $11 \mu \mathrm{m}$, collectively shown as D1 and D2 in Fig. 6(a), were connected to the differential inductor $L_{\text {diff }}$ to form a resonator.

As shown in Fig. 7, multiple small varactors were used instead of two large varactors to minimize eddy current loss. We used the EM simulator to optimize the size, the spacing, and the location of these varactors inside an inductor. However, unlike metal fills, varactors actively participate in the circuit operation and carry AC current. Their effect on the inductor is thus potentially more complicated than just extra loss due to eddy currents. On the other hand, it is not possible to conduct EM simulations that include varactor device behavior. So for EM simulations, only the eddy current effect was taken into account, and the varactors were replaced with parallel plate capacitors with similar plate resistivity as the varactors while the wiring was kept the same. From EM simulations it was determined that the size of the varactors can be made larger than the size of the metal fills because the varactors are made from $\mathrm{p}+$ base in the $\mathrm{n}$-well which has higher resistivity than metal. From (5), we know that the higher resistivity material will have less eddy current loss in them and therefore a larger surface area is tolerable. In fact, the large amount of low-resistive wiring required to connect the varactors together dominates the eddy current loss in the inductor. 


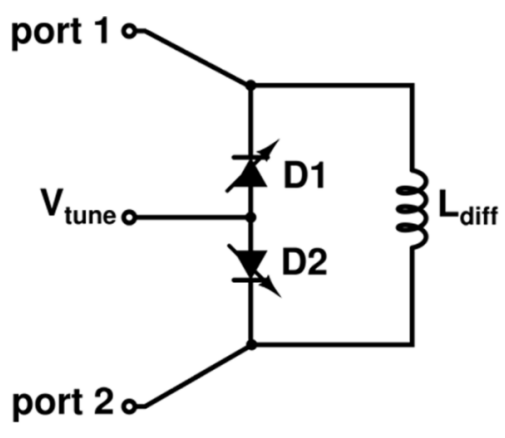

(a)

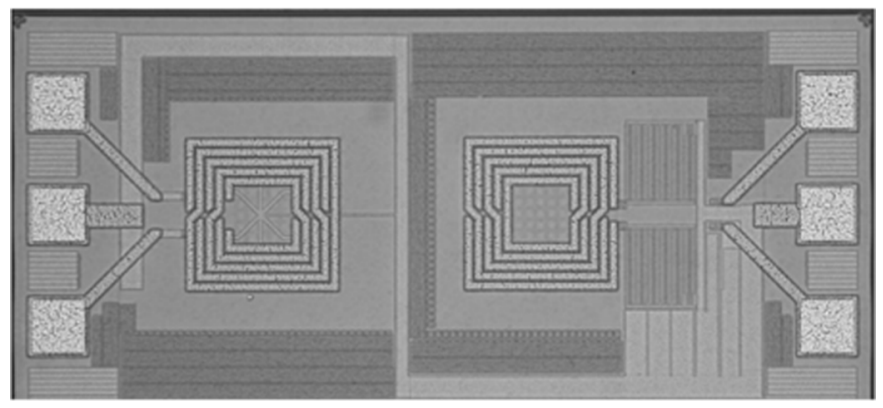

(b)

Fig. 6. (a) Resonator schematic. (b) Die photo: Resonator IN (left) and Resonator OUT (right).

(a) Traditional

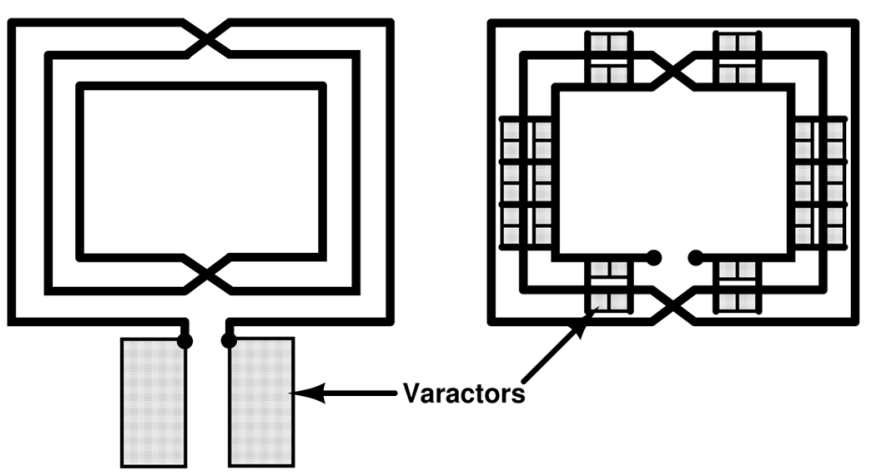

Fig. 7. (a) Standard differential inductor layout for Resonator OUT. (b) Inductor layout used for Resonator IN.

Appropriate layout techniques have to be used to minimize this loss, as discussed next.

We first modified the layout of the differential inductor $L_{\text {diff }}$ to allow placement of the varactors inside the inductor. In a traditional differential inductor layout, shown in Fig. 7(a), the inductor leads are on the outermost turn of the inductor since the varactors are placed outside of the inductor. By folding the leads of the inductor "outside in," as shown in Fig. 7(b), the cathodes of the varactors can be connected along the innermost turn of the inductor. Although this connection can result in a distributed effect, which is undesirable in a narrow-band circuit, the actual effect on the circuit is small since the innermost turn only contributes to a small fraction of the total inductance. A simulation

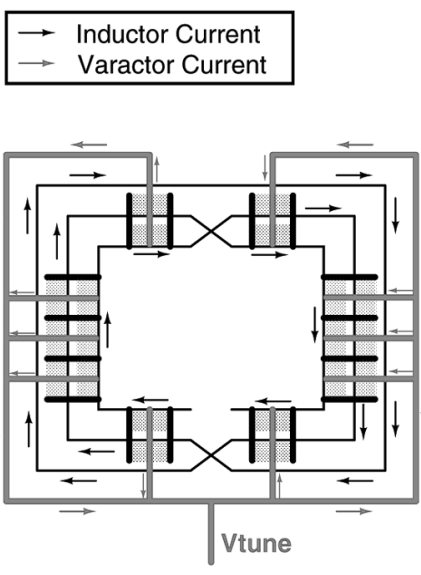

(a)

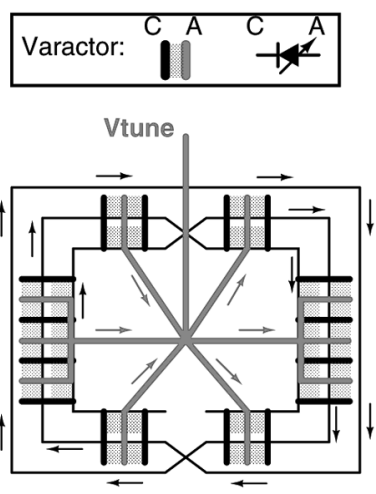

(b)
Fig. 8. Two layout styles for the $V_{\text {tune }}$ line and the associated current flows.

using a distributed model for the inner section of the inductor, connected to varactors, confirmed that the distributed effect is indeed negligible.

Fig. 8 shows two possible layout configurations for the $V_{\text {tune }}$ line that connects the anodes of the varactors and at the same time avoids any closed loops.

In Fig. 8(a), the anode of the varactors are connected on the outside of the inductor. The drawback of this configuration is that the currents in large sections of the $V_{\text {tune }}$ line are parallel to the flow of the inductor current, causing unwanted magnetic coupling in the wires. The configuration shown in Fig. 8(b) distributes the $V_{\text {tune }}$ line from the center of the inductor, thus keeping the wires with parallel current flow far apart. Although there is possible coupling between the components themselves and the inductor in addition to the coupling between the wiring and the inductor, we did not observe any degradation in the performance of the circuit due to such coupling. Fig. 9(a) shows the details of the rake-shaped metal wiring used to connect the anodes of the varactors. The shape of the wiring is similar to that of a patterned ground shield (PGS) [10]. The fingers of the rake-shaped wiring are oriented such that the current flow in them is perpendicular to the direction of the inductor current to minimize magnetic coupling between the two. Furthermore, since the $V_{\text {tune }}$ node in Fig. 6(a) is a signal ground for differential signals, the rake-shaped wiring acts as a grounded PGS which absorbs any stray electric field from the inductor to the substrate, thus improving the $Q$ of the inductor [10].

Besides the size of the varactors and their interconnect, the placement of these varactors is also important. Simulations showed that the $Q$ degradation is minimal when the varactors are placed under the inductor traces instead of at the center of the inductor. Not only are they exposed to a smaller magnetic field there, but they also perform the role of a PGS by further electrically isolating the inductor from the lossy substrate.

Two resonators were constructed, one with varactors inside the inductor, henceforth referred to as Resonator IN, and another with varactors outside the inductor, henceforth referred to as Resonator OUT. The die photo of the test structure is shown 


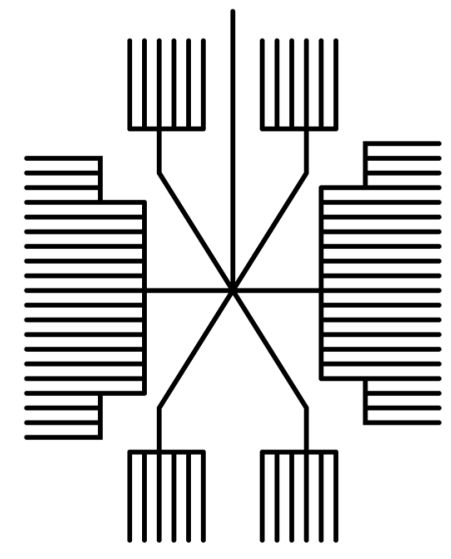

(a)

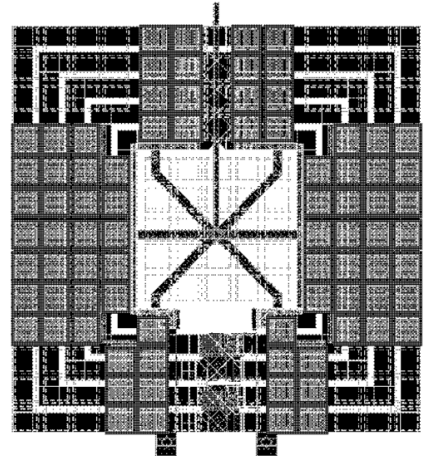

(b)
Fig. 9. (a) Rake-shaped $V_{\text {tune }}$ line. (b) Resonator IN layout.

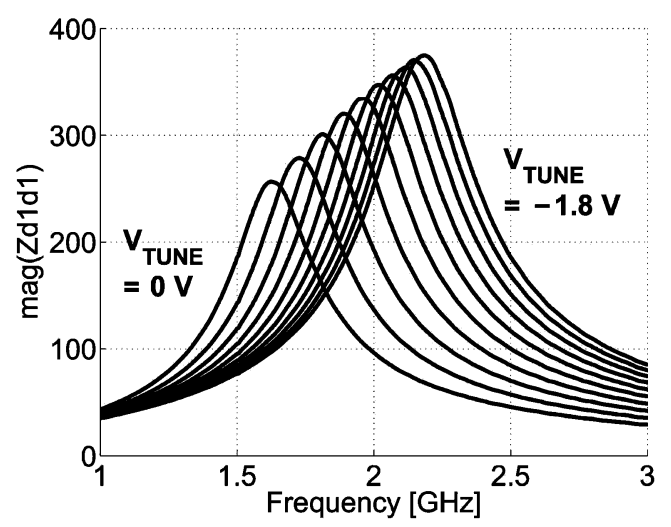

Fig. 10. Magnitude of the differential impedance $Z_{d 1 d 1}$ of Resonator IN for different tuning voltages $V_{\text {tune }}$.

in Fig. 6(b). The resonators were probed using an SGS probe ${ }^{1}$ and the $S$-parameters were extracted using an Agilent N5230A network analyzer. The varactors were tuned through bias-tees connected to the two signal paths of the SGS probe. The differential $S$-parameter $S_{d 1 d 1}$ was calculated from

$$
S_{d 1 d 1}=\frac{1}{2}\left(S_{11}+S_{22}-S_{12}-S_{21}\right)
$$

from which we derived the differential $Z$-parameter $Z_{d 1 d 1}$

$$
Z_{d 1 d 1}=2 Z_{o}\left(\frac{1+S_{d 1 d 1}}{1-S_{d 1 d 1}}\right)
$$

where $Z_{o}$ is the characteristic impedance, equal to $50 \Omega$.

Fig. 10 shows the measured magnitude of $Z_{d 1 d 1}$ versus frequency for Resonator IN for different tuning voltages $V_{\text {tune. }}$. As

\footnotetext{
${ }^{1}$ In the resonator shown in Fig. 6(a), the anodes of the varactors ( $\left.V_{\text {tune }}\right)$ are connected to ground, and the varactors are tuned from the cathode side (ports 1 and 2) through bias-tees. This bias configuration was used because it is not possible to apply a DC voltage to the ground pin of an SGS probe. While in a typical $\mathrm{VCO}$, the cathodes of the varactors are biased at $V_{\mathrm{dd}}$ through the center-tap of the inductor, and the varactors are tuned from the anode side. The only difference between the two configurations is the sign of the tuning curve $(K V)$ slope.
}

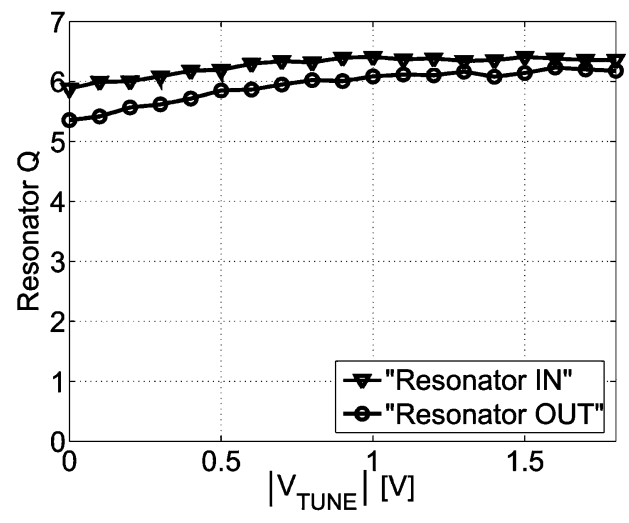

Fig. 11. Comparison of the quality factor $Q$ of Resonator IN and Resonator OUT.

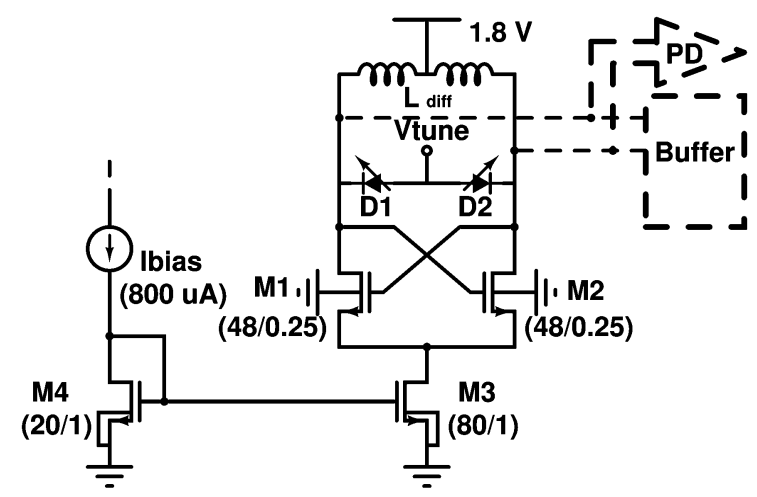

Fig. 12. Schematic for the VCO-in-the-coil and regular VCO; the buffer and peak detector (PD) are auxiliary circuits to facilitate the measurements.

expected, these curves have the characteristic shape of a parallel $L C$ tank impedance.

From EM simulation of $L_{\text {diff }}$ by itself, we found inductor quality factor $Q_{L}$ of about 7 at $2 \mathrm{GHz}$. The varactors have a simulated quality factor $Q_{C}$ of about 40 at $2 \mathrm{GHz}$. The combined $Q$ of the tank [9], which is equal to $\left(Q_{L}^{-1}+Q_{C}{ }^{-1}\right)^{-1}$, is thus dominated by the quality factor of the inductor. Any degradation in the quality factor of the tank gives us an indication of the degradation in the quality factor of the inductor due to the varactors and wiring underneath it.

One way to define the $Q$ of the tank is center frequency divided by $3-\mathrm{dB}$ bandwidth. Fig. 11 shows the $Q$ calculated this way using the curves shown in Fig. 10 for both Resonator IN and Resonator OUT. The $Q$ of Resonator IN is slightly higher than that of Resonator OUT due to the electrical shielding effect of the $V_{\text {tune }}$ wiring.

The resonator experiment shows that a tank circuit built with varactors underneath the inductor is tunable and has a comparable $Q$ to a tank with traditional layout.

\section{VCO-IN-THE-COIL}

Constructing a resonator circuit with sufficient $Q$ is a critical step in designing a $\mathrm{VCO}$, and adding active circuitry to maintain oscillation while keeping noise to a minimum is the other half of the challenge. We used a standard VCO architecture shown in Fig. 12. 


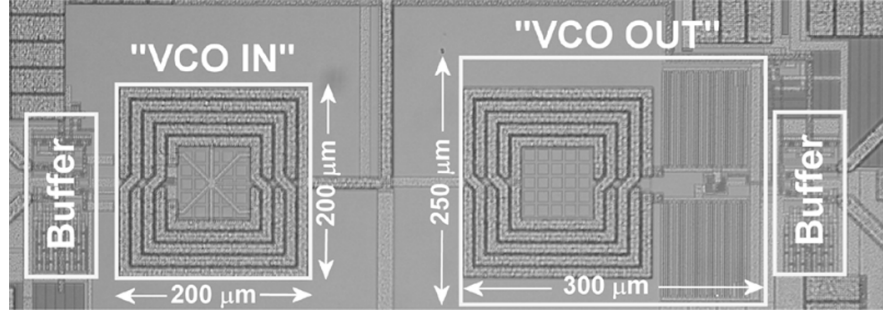

Fig. 13. VCO die photo.
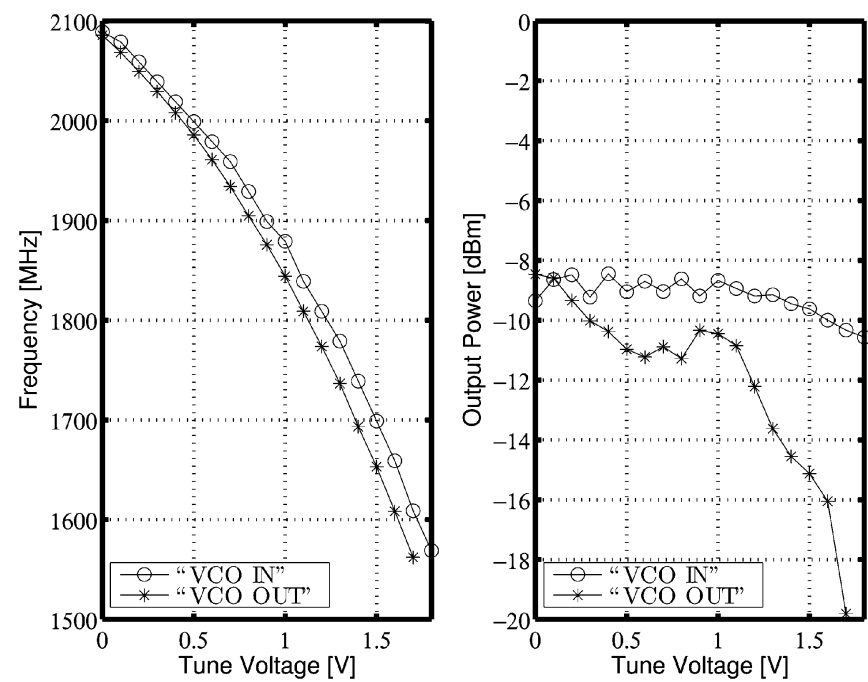

Fig. 14. Measured tuning characteristic of the VCOs (left) and the output power of the VCOs (right).

An important concern in the VCO layout is the resistance of the $V_{\text {tune }}$ line, since its series resistance adds thermal noise which is directly converted into phase noise [9]. Parasitic resistance extraction was performed on the longest wire path from the $V_{\text {tune }}$ pin to the varactor anode. From simulation, the lowest series resistance tolerable for the phase noise requirement was calculated. In order to lower the wire resistance, multiple metal layers were strapped together. Lowering the resistance of the $V_{\text {tune }}$ line worsens its eddy current effect on the inductor, as (5) suggests. However, the rake-shaped wiring in Fig. 9(a) prevents eddy currents from circulating in large loops, thus minimizing their effect.

Fig. 9(b) shows the complete layout of the VCO. The active devices, which include a cross-coupled pair, current source, and current mirror were placed at the bottom center of the inductor. Like the varactors, we can only treat the transistors as static elements, and simulate their eddy current effect on the inductor. Any dynamic coupling between the transistors and the inductor cannot be measured easily. However for the VCO, the transistors and the inductor are part of the same circuit and carry the same signals, therefore the parasitic coupling as demonstrated in [11] is not a concern. We ran EMX simulations on the entire VCO structure which includes the differential inductor, the rake-shaped multi-layer metal routing and other metal and polysilicon interconnects for the varactors and active devices. The diffusions in the varactors and the active devices are modeled by adding special interconnect layers with the appropriate
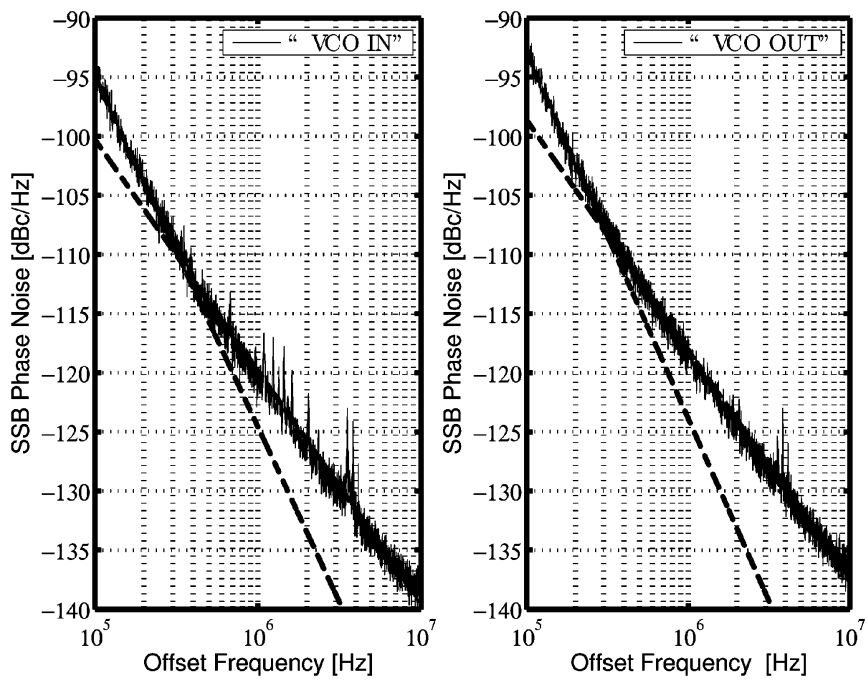

Fig. 15. Measured phase noise spectrum for a $2 \mathrm{GHz}$ carrier frequency; the dashed lines have a $1 / f^{2}$ and $1 / f^{3}$ slope, the corner frequency is $\sim 300 \mathrm{kHz}$.

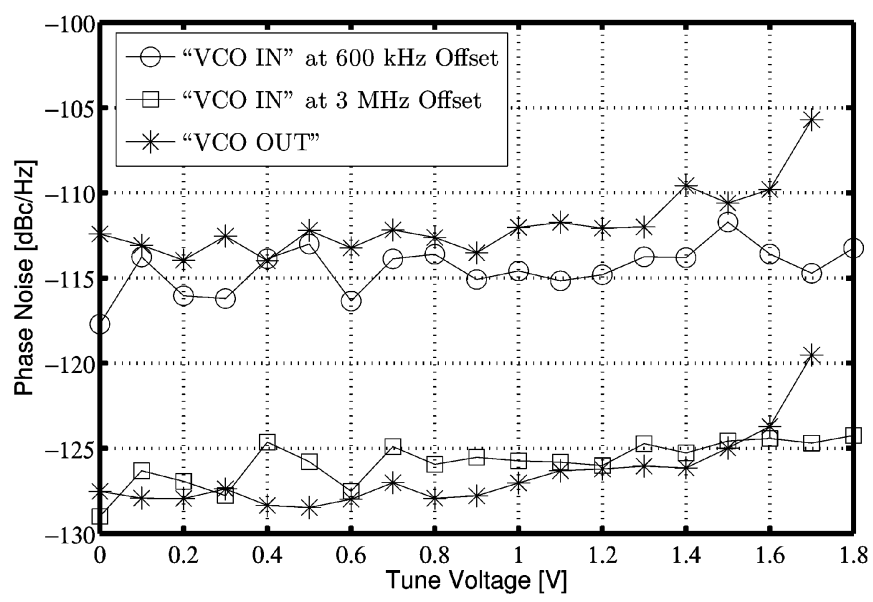

Fig. 16. Measured phase noise for different tuning voltages.

resistivity and position in the layer stack. The simulated resonator $S$-parameters, which include all the eddy current effects, were combined with active device models to simulate the VCO functionality and performance in circuit simulations. Simulation results confirmed that the performance of the compact VCO is very close to the performance of the $\mathrm{VCO}$ with a plain inductor.

Two VCOs were constructed: the VCO-in-the-coil with varactors and actives inside the inductor, henceforth referred to as VCO IN, and a regular VCO with varactors and actives outside the inductor, henceforth referred to as VCO OUT. The CMOS VCOs were implemented in a $0.25-\mu \mathrm{m}$ BiCMOS process with only the peak detector implemented in bipolar. The on-chip peak detector [12] is used for calibrated amplitude measurements without requiring the calibration of the output buffer. Both VCOs, shown in Fig. 13, consume $3.2 \mathrm{~mA}$ from a 1.8-V supply. The VCO output is connected to a buffer stage as well as a peak detector running off a $2.5-\mathrm{V}$ supply.

Four sets of VCOs were characterized with a Cascade RF probe station and an Agilent E4446A spectrum analyzer; the measured data was very consistent and the results of a typical set are shown in Figs. 14-16. Fig. 14(a) shows the tuning characteristic of the VCOs; a very wide tuning range 
of $520 \mathrm{MHz}$, or $26 \%$ of the center frequency, was achieved. VCO IN uses an outside-in inductor layout while VCO OUT uses a regular inductor layout which results in slightly different inductance values; this explains the small frequency difference seen in Fig. 14(a). The inductor in VCO OUT does not have the rake-shaped $V_{\text {tune }}$ wiring and does not benefit from its PGS effect. However, in simulation we did not observe a significant difference in inductance between a plain inductor and one with PGS, therefore, we do not believe that the frequency difference is due to the electrical shielding effect of the varactors and wiring. Fig. 14(b) shows the output power for different tuning voltages. Both oscillators have a similar output power except for high tuning voltages, which corresponds to low output frequencies. We currently do not have a conclusive explanation for the higher output power from VCO IN and the reduction of output power in VCO OUT. Fig. 15 shows the noise spectrum of the VCOs and Fig. 16 shows the variation in phase noise for different tuning voltages at various offset frequencies. The two VCOs have close to identical performance.

Returning to the die photo of the VCOs in Fig. 13, VCO OUT on the right occupies $0.3 \times 0.25 \mathrm{~mm}^{2}$, and VCO IN on the left occupies $0.2 \times 0.2 \mathrm{~mm}^{2}$, resulting in an area saving of $47 \%$.

\section{DISCUSSION AND CONCLUSION}

We have shown through experiments and simulations that eddy current loss accounts for the majority of the degradation in quality factor when devices or metal fills are placed inside the inductor. However, by breaking the devices into smaller pieces, the eddy current loops are localized and therefore the resistive loss is minimized. By avoiding current loops in the wiring that connects the devices together and to the inductor, the additional eddy current loss in the wiring is reduced. Furthermore, unwanted magnetic coupling between the inductor current and the device currents is reduced by keeping their paths perpendicular wherever possible. Using these techniques, we demonstrated that large numbers of metal fills or varactors and active devices can be placed underneath an inductor without a significant degradation in its $Q$.

This circuit-in-the-coil technique can be implemented in many layouts without extra post-processing steps and can be further extended to other applications. For example, the capacitors of a phase-locked loop (PLL) filter typically occupy a large amount of area. They can be placed under the VCO inductor without a significant risk of spurious signal coupling since the $V_{\text {tune }}$ line is already connected to the VCO. The capacitors for tail current filters to reduce phase noise in VCOs [13] usually consume a significant area and could also take advantage of this compact layout technique. Supply and other decoupling capacitors, when properly laid out and interconnected can also be placed under the spiral inductors. However, such configurations must consider all possible unwanted coupling between the inductor and the circuitry connected to the decoupled lines.

\section{ACKNOWLEDGMENT}

The authors would like to thank Philips Semiconductors for chip fabrication, S. Kapur and D. Long of Integrand Software for the use of the EMX simulation tool, B. Soltanian for the VCO buffer, peak detector design, and help with measurements, and S. Chatterjee for technical discussions.

\section{REFERENCES}

[1] T. H. Lee, The Design of CMOS Radio-Frequency Integrated Circuits, 1st ed. Cambridge, U.K.: Cambridge Univ. Press, 1998

[2] R. Ahola et al., "A single-chip CMOS transceiver for $802.11 \mathrm{a} / \mathrm{b} / \mathrm{g}$ wireless LANs," IEEE J. Solid-State Circuits, vol. 39, no. 12, pp. 2250-2258, Dec. 2004.

[3] F. Zhang, C.-F. Chu, and P. Kinget, "Voltage-controlled oscillator in the coil," in Proc. IEEE Custom Integrated Circuits Conf., Sep. 2005, pp. 587-590.

[4] C. Detcheverry, "The effect of copper design rules on inductor performance," in Proc. Eur. Solid-State Device Research Conf., Sep. 2003, pp. $107-110$.

[5] C.-L. Chen, "Effects of CMOS process fill patterns on spiral inductors," Microw. Opt. Technol. Lett., vol. 36, pp. 462-465, 2003.

[6] W. B. Kuhn et al., "Spiral inductor performance in deep-submicron bulk-CMOS with copper interconnects," in IEEE MTT-S Int. Microwave Symp. Dig., Jun. 2002, vol. 1, pp. 301-304.

[7] D. Halliday, R. Resnick, and K. S. Krane, Physics, 4 ed. New York: Wiley, 1992, vol. 2

[8] S. Kapur and D. E. Long, "Large-scale full-wave simulation," in Proc. Design Automation Conf., Jun. 2004, pp. 806-809.

[9] P. Kinget, "Integrated GHz voltage controlled oscillators," in Analog Circuit Design: (X)DSL and Other Communication Systems; RF MOST Models; Integrated Filters and Oscillators, W. Sansen, J. Huijsing, and R. van de Plassche, Eds. Boston, MA: Kluwer, 1999, pp. 353-381.

[10] C. P. Yue and S. S. Wong, "On-chip spiral inductors with patterned ground shields for Si-based RF ICs," IEEE J. Solid-State Circuits, vol. 33, no. 5, pp. 743-752, May 1998.

[11] Y. Papananos and N. Nastos, "Inductor over MOSFET: Operation and theoretical study of a CMOS RF three-dimensional structure," in Proc. 24th Int. Conf. Microelectronics, May 2004, pp. 525-529.

[12] R. G. Meyer, "Low-power monolithic RF peak detector analysis," IEEE J. Solid-State Circuits, vol. 30, no. 1, pp. 65-67, Jan. 1995.

[13] E. Hegazi, H. Sjoland, and A. A. Abidi, "A filtering technique to lower LC oscillator phase noise," IEEE J. Solid-State Circuits, vol. 36, no. 12, pp. 1921-1930, Dec. 2001.

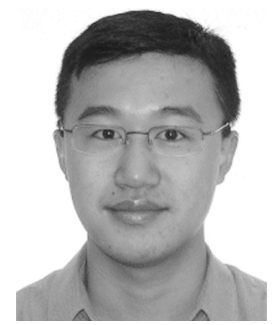

Frank Zhang (S'00) received the B.E. and M.S degrees in electrical engineering from the Cooper Union for the Advancement of Science and Art, New York, NY, in 2000 and 2001, respectively. Currently, he is working toward the Ph.D. degree at Columbia University, New York, NY.

From 2001 to 2003 , he was an intern at Motorola's Wireless Integrated Technology Center, South Plainfield, NJ. His current research interests include analog and RF integrated circuits.

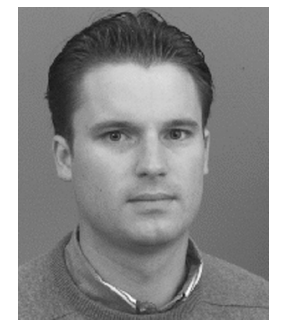

Peter R. Kinget (S'88-M'90-SM'02) received the engineering degree (summa cum laude) in electrical and mechanical engineering and the Ph.D. degree (summa cum laude) in electrical engineering from the Katholieke Universiteit Leuven, Belgium, in 1990 and 1996, respectively.

From 1991 to 1995 , he received a graduate fellowship from the Belgian National Fund for Scientific Research (NFWQ) to work as a Research Assistant at the ESAT-MICAS Laboratory of the Katholieke Universiteit Leuven. From 1996 to 1999, he was at Bell Laboratories, Lucent Technologies, Murray Hill, NJ, as a Member of Technical Staff in the Design Principles Department. From 1999 to 2002, he held various technical and management positions in IC design and development at Broadcom, CeLight, and MultiLink. In the summer of 2002, he joined the faculty of the Department of Electrical Engineering, Columbia University, New York, NY. His research interests are in analog and RF integrated circuits and signal processing. He has published over 50 papers in journals and conferences and holds three U.S. patents with several applications under review. His research group has received funding from the National Science Foundation, the Semiconductor Research Corporation, an IBM Faculty Award, and from several grants from semiconductor companies.

Dr. Kinget has served on the Technical Program Committee of the IEEE Custom Integrated Circuits Conference (CICC) and the Symposium on VLSI Circuits. He currently serves on the Technical Program Committee of the European Solid-State Circuits Conference, and the IEEE International Solid-State Circuits Conference. He has been an Associate Editor for the IEEE JOURNAL OF SOLID-STATE CIRCUITS since 2003. 